\title{
ON CHAIN VARIETIES OF LINEAR ALGEBRAS
}

\author{
BY
}

\section{A. ARTAMONOV}

ABSTRACT. In the present paper we study varieties of linear $k$-algebras over a commutative associative Noetherian ring $k$ with 1 , whose subvarieties form a chain. We describe these varieties in terms of identities in the following cases: residually nilpotent varieties, varieties of alternative, Jordan and $(-1,1)$-algebras.

1. Introduction. In [1] we have already described homogeneous chain varieties of linear algebras over a field. Recall that variety is homogeneous iff its free algebras are graded relative to the degree-function. If the ground field is infinite, then any variety of algebras over this field is homogeneous. In the second paper [2] we gave the description of chain varieties of restricted $p$-algebras Lie over an infinite field.

In the present paper we describe in Theorems 2-15 chain varieties $V$ of linear $k$-algebras, $k$-commutative associative Noetherian ring with 1 , in the following cases:

(i) if $G$ is a free algebra in $V$ then $\bigwedge_{n} G^{n}=0$ (this is always valid when $V$ is locally nilpotent), i.e. $V$ is residually nilpotent,

(ii) $V$ is a variety of alternative algebras,

(iii) $V$ is a variety of $(-1,1)$-algebras,

(iv) $V$ is a variety of Jordan algebras.

Throughout the paper $k$ is a fixed commutative associative ring with 1 , and $V$ is a variety of $k$-algebras with chain of subvarieties. We also assume that Ann $V=\{\alpha \in k \mid(\forall A \in V)(\alpha A=0)\}=0$.

Finally it is worthy of note that similar problems for associative rings were studied in [3], [4], [5].

2. Residually nilpotent varieties. Let

$$
f\left(x_{1}, \ldots, x_{n}\right)=\sum_{i=1}^{d} f_{i}=0
$$

be an identity in $V$ and $x_{1}$ appears $i$ times in $f_{i}$. Then for any $\alpha_{1}, \ldots, \alpha_{d} \in$ $k$, (1) implies 


$$
\alpha_{1} \cdots \alpha_{d} w\left(\alpha_{1}, \ldots, \alpha_{d}\right) f_{i}=0
$$

where $W(, \ldots$,$) is the Vandermonde determinant. Thus, as usual (see [1], [6]),$ we have

PROPOSITION 1. If $k$ has a unique maximal ideal $\mathfrak{m}$, i.e. $k^{*}=k \backslash \mathfrak{m}$, then if $\bar{k}=k / \mathfrak{m}$ is infinite, (1) is equivalent to the set of identities $f_{i}=0, i=1, \ldots, d$. If $\bar{k}=\mathbf{F}_{q}$, then $f=0$ is equivalent to the set of identities

$$
\sum_{i \equiv t \bmod (q-1)} f_{i}=0, \quad t=1, \ldots, q-1,
$$

and for all $\alpha \in \mathfrak{m}$,

$$
\alpha^{d(d-1) / 2} f_{i}=0 .
$$

To prove the last affirmation take $\alpha_{i}=1+\alpha^{i} \in k^{*}$. Then by (1')

$$
\begin{aligned}
W\left(\alpha_{1}, \ldots, \alpha_{d}\right) & = \pm \prod_{r<s}\left(\alpha^{r}-\alpha^{s}\right)= \pm \alpha^{d(d-1) / 2} \prod_{r<s}\left(1-\alpha^{s-r}\right) \\
& =\delta \alpha^{d(d-1) / 2}, \quad \delta \in k^{*} .
\end{aligned}
$$

THEOREM 1. Let $V$ be a variety of $k$-algebras with chain of subvarieties $k$ as in Proposition 1. If $V$ is homogeneous then either $x y=0$ in $V$, or $m=0$ and $V$ was described in [1]. If $\bar{k}=k / \mathrm{m}$ is infinite, then $V$ is homogeneous.

Proof. By Proposition $1, V$ is homogeneous if $\bar{k}=k / \mathrm{m}$ is infinite. Suppose that $V$ is homogeneous and $x y=0$ in $V$ implies $m x=0$. Then by homogeneity $\mathfrak{m} x=0$ in $V$. If $m x=0$ implies $x y=0$, then $V$ has an identity $x y=$ $\alpha x y+\beta y x$, where $\alpha, \beta \in \mathfrak{m}$. Since $1-\alpha \in k^{*}$ we can suppose that $\alpha=0$. Therefore, $x y=\beta y x$ and $(1-\beta) x^{2}=0=x^{2}$, i.e. $x y+y x=0$. Eventually $x y=\beta y x=$ $-\beta x y$ and $(1+\beta) x y=0=x y$.

Assume now that $V$ is a residually nilpotent variety, i.e. in a $V$-free algebra $G$ with free generators $x_{i}, i=1,2, \ldots$,

$$
\bigwedge G^{i}=0
$$

where $G^{i}=\left(\Sigma g_{1} \cdots g_{i}\right.$ with different bracketing $\left.g_{j} \in G\right)$. Note that in this case (2) is valid for any $V$-free algebra.

Let $F$ be a $V$-free algebra with one free generator $x$. Let $I_{n}=$ Ann $F / F^{n}$ $\triangleleft k, k_{n}=k / I_{n}$. Note that $F / F^{2}=k_{2} x$ with zero multiplication. Thus there is a one-to-one correspondence between ideals of $k_{2}$ and subvarieties of var $F / F^{2}$ consisting of all $k_{2}$-modules, annihilated by given ideal of $k_{2}$. Thus $k_{2}$ has a unique maximal ideal $\mathrm{m}_{2}$. Let $\mathrm{m}$ be the inverse image of $\mathrm{m}_{2}$ in $k$. Define $\mathrm{m}_{j}=$ $\mathfrak{m} / I_{j}$. If $\beta \in k_{n} \backslash \mathrm{m}_{n}$, then for some $\gamma \in k_{n}$ we have $\beta \gamma \equiv 1 \bmod I_{2} / I_{n}$ and since 
$I_{2}^{n} \subseteq I_{n}, \beta \gamma^{\prime}=1$ in $k_{n}$ for some $\gamma^{\prime} \in k_{n}$. Hence $k_{n}$ is a ring with unique maximal ideal $m_{n}$.

Now for each $\beta \in k$ the ideal $\beta F$ is verbal, so since $m$ is finitely generated, $\mathrm{m} F=\alpha F$ for some $\alpha \in \mathrm{m}$. In $V$ either $x^{2}=0$ implies $\alpha x=0$, or $\alpha x=0$ implies $x^{2}=0$. Thus in $F$ either

$$
x^{2}=\alpha \beta_{1} x+\alpha \beta_{2} x^{2}+\sum_{i=3}^{d} \alpha g_{i}(x), \quad \text { or } \quad \alpha x=\sum_{i=2}^{d} g_{i}(x)
$$

where $g_{i}(x)$ is a sum of monomials of degree $i$. By (3) and Proposition 1 we have in $F_{n}$

$$
\alpha^{t} x^{2}=0, \quad t=1+1 / 2 d(d-1),
$$

where $t$ does not depend on $n$. Hence $\alpha^{t} x^{2} \in \bigwedge_{n} F_{n}=0$ and, therefore, $m^{t} F^{2}$ $=\alpha^{t} F^{2}=0$.

Consider now $k_{n}$-module $F / F^{n}, n>1$. Note that $m_{n}$ is the Jacobson radical of $k_{n}$. Thus, by Theorem 4.2 [7, p. 12], $\bigwedge_{r} m_{n}^{r} F / F^{n}=0$. Hence, for some $r>1$ we have $\mathrm{m}_{n}^{r} F / F^{n} \subseteq F^{2} / F^{n}$ and $\mathrm{m}_{n}^{r+t} F_{n} \subseteq \mathrm{m}_{n}^{t} F_{n}^{2}=0$ by (3'), where $F_{n}=$ $F / F^{n}$. Thus, $m^{r+t} F \subseteq F^{n}$ and $m^{r+t} F \subseteq \bigwedge_{n} F^{n}=0$. So $m$ is nilpotent and $k / m=k_{2} / m_{2}=\bar{k}$ is a field. Finally we have

Proposition 2. $k$ has a unique maximal nilpotent ideal $m, m^{d}=0$.

Now by Theorem 1 without loss of generality we can assume that $\bar{k}=$ $k / m=F_{q}$.

Let $G$ be a $V$-free algebra with free generators $x_{i}, i>1$,

$$
G^{t}=\left\{\sum g_{1} \cdots g_{t} \text { with different bracketing } g_{i} \in G\right\} \text {. }
$$

Put

$$
\hat{G}=\bigoplus_{i \geq 1} G^{i} / G^{i+1}
$$

Since $G^{i} G^{j} \subseteq G^{i+j}$ we can define in $\hat{G}$ a multiplication

$$
\left(x+G^{i+1}\right)\left(y+G^{j+1}\right)=x y+G^{i+j+1},
$$

$x \in G^{i}, y \in G^{j}$. It is clear that $G$ is generated by $y_{i}=x_{i}+G^{2}, i>1$.

Proposition 3. $\hat{G}$ is a relatively free $k$-algebra with free generators $y_{i}$, $i \geqslant 1$. $\operatorname{var} \hat{G}$ has a chain of subvarieties.

ProOF. Since $\hat{G}$ is homogeneous we need to prove that if $\boldsymbol{g}=$ $g\left(X_{1}, \ldots, X_{n}\right)$ is a homogeneous polynomial of degree $d$, then $g\left(y_{1}, \ldots, y_{n}\right)=$ 0 implies $g\left(v_{1}, \ldots, v_{n}\right)=0$ for all $v_{i} \in \hat{G}$. Note that

(4) $g\left(y_{1}, \ldots, y_{n}\right)=0 \Longleftrightarrow g\left(x_{1}, \ldots, x_{n}\right) \in G^{d+1} \Longleftrightarrow g\left(v_{1}, \ldots, v_{n}\right)=0$ 
for all homogeneous $v_{i} \in \hat{G}$. Thus, if $g$ is multilinear, then $g=0$ is an identity in $\hat{G}$. Put $D=D(g)=d-n$. So, we have already proved the proposition in the case $D=0$. Suppose that $D>1$; then, for example,

$$
\begin{aligned}
h & =h\left(x_{1}, x^{\prime}, x_{2}, \ldots, x_{n}\right) \\
& =g\left(x^{\prime}+x_{1}, x_{2}, \ldots, x_{n}\right)-g\left(x_{1}, \ldots, x_{n}\right)-g\left(x^{\prime}, x_{2}, \ldots, x_{n}\right)
\end{aligned}
$$

has $D^{\prime}=D(h)<D=D(g)$. Note that by (4) $h\left(y_{1}, \ldots, y_{n+1}\right)=0$ and, hence, by induction, $h=0$ is an identity in $\hat{G}$. Eventually, if $g\left(v_{1}, \ldots, v_{n}\right)=$ $g\left(v^{\prime}, v_{2}, \ldots, v_{n}\right)=0$, then by $\left(4^{\prime}\right), g\left(v_{1}+v^{\prime}, v_{2}, \ldots, v_{n}\right)=0$. Thus, $\hat{G}$ is relatively free.

Now if $f, g$ are homogeneous in $\hat{G}$ the verbal ideals $\{f\},\{g\}$ generated by $f$ and $g$ in $G$ are comparable, i.e., for example,

$$
g=g\left(f\left(\cdots, u_{j}\left(\cdots x_{i} \cdots\right), \cdots\right), \cdots, v_{j}\left(\cdots x_{i} \cdots\right), \cdots\right)
$$

and, hence, in $\hat{G}$,

$$
g=g\left(f\left(\cdots, u_{j}\left(\cdots y_{i} \cdots\right), \cdots\right), \cdots, v_{j}\left(\cdots y_{i} \cdots\right), \cdots\right),
$$

i.e. $f=0$ implies $g=0$. Since in [1] we used only this criterion for the description, var $\hat{G}$ has a chain of subvarieties. This completes the proof.

Now by Theorem 1 since $\hat{G}$ is homogeneous either $\hat{G}^{2}=0$, i.e. $G^{2}=G^{3}$ and $G^{2} \in \bigwedge G^{n}=0$, or $\hat{G}$ is an $\mathrm{F}_{q}$-algebra, $m G=\alpha G \subseteq G^{2}$ and $\hat{G}$ satisfies identities from [1, Theorems 2-8]. Our aim is to prove that very often $\hat{G}=G$. Note that if $G_{n}$ is a $V$-free algebra of rank $n$, that is, $G_{n}$ possesses free generators $x_{1}, \ldots, x_{n}$, then $G_{n}^{r}=G_{n}^{r+1}$ implies $G_{n}^{r}=0$.

THEOREM 2. If $\hat{G}$ is commutative, associative, $G^{2} \neq 0$, and either $x^{p}=0$, $p=$ char $F_{q}$, or $x_{1} \cdots x_{p+1}=0$, then $G$ has the same property. If $x^{p}=0$, then $\mathfrak{m}=0$. If $x_{1} \cdots x_{p+1}=0$ and $\mathfrak{m} \neq 0$ then $x^{p}=\alpha x, q=p, \mathfrak{m}=\mathrm{F}_{p} \alpha, \alpha^{2}=$ $0, \alpha x^{2}=0$. In all the cases $V=\operatorname{var} G$ has $a$ chain of subvarieties.

Proof. If $x^{p}=0$ in $\hat{G}$, then $G_{1}^{p}=G_{1}^{p+1}$ and, as we have already noticed, this implies $G_{1}^{p}=0$. Similarly, $G_{2}^{2 p-1}=G_{3}^{3 p-2}=0$ and by Proposition $1, G$ is commutative, associative, $x^{p}=0$. The case $x_{1} \cdots x_{p+1}=0$ is analogous.

Now if $G^{2} \neq 0$ then $\alpha G \leqslant G^{2}$ and if $x^{p}=0$, by Proposition $1, \alpha x=0$, i.e. $m=0$. If $m \neq 0$ and $x_{1} \cdots x_{p+1}=0$, then by Proposition $1, \alpha x=\beta x^{p}$, $\beta \in k, p=q$. Multiplying by $x$ we obtain $\alpha x^{2}=\beta x^{p+1}=0$ and, therefore, $\alpha^{2} x=\alpha \beta x^{p}=0$, i.e. $\alpha^{2}=0$. So, if $\alpha \neq 0$, then $\beta \in k^{*}$, and without loss of generality we can assume $\beta=1$. In this case $V$ has a chain of subvarieties

$$
0 \subset\left\{x_{1} x_{2}=0\right\} \subset \cdots \subset\left\{x_{1} \cdots x_{p}=0\right\} \subset\left\{x^{p}=\alpha x=0\right\} \subset V .
$$

Theorem 2 is proved. 
Suppose now that $\hat{G}$ is a metabelian Lie algebra. Then $\hat{G}$ satisfies conditions of [1, Theorem 3]. Starting from now we are going to use the following notation

$$
x_{1} \cdots x_{n}=\left(\left(\cdots\left(\left(x_{1} x_{2}\right) x_{3}\right) \cdots\right) x_{n-1}\right) x_{n}, \quad x y^{n}=x y \cdots y .
$$

THEOREM 3. If $\hat{G}$ is a metabelian Lie algebra from $\left[1\right.$, Theorem 3], $G^{2} \neq$ 0 , then $m=0$ and $G$ is either metabelian Lie algebra, $\hat{G}=G$, with one of the following sets of identities $\left(p=\right.$ char $\left.\mathbf{F}_{q}\right)$ :

(i) $x y z^{p}=x y^{p} x^{p-1}=0$,

(ii) $x_{1} \cdots x_{p+3}=0$ and if $p=2$ then either $y x^{2} y=0$ or yxzt $+z x y t+$ txyz $=0$; if $p=q>3$ then either $x y^{p+1}=0$ or $x y z^{p}+y z x^{p}+z x y^{p}=0$, or $p=q \geqslant 3, x_{1} \cdots x_{p+3}=0$ and $G$ satisfies one of the following sets of identities:

(i) $p=q \geqslant 3, x y^{p+1}=0, x^{2}=0, J(x, y, z)=\beta x y z^{p}, \beta \in \mathrm{F}_{p}^{*},(x y)(z t)=0$,

(ii) $p=q=3, x^{2}=(x y)(z t)=0, J(x, y, z)=\beta\left(x y z^{p}+y z x^{p}+z x y^{p}\right)$.

In all the cases $V$ has a chain of subvarieties.

Proof. Since $x^{2}=0$ in $\hat{G}, \hat{G}_{1}^{2}=G_{1}^{2}=0$, i.e. $x^{2}=0$ in $V$. As we have already noticed above, $m G_{1} \subseteq G_{1}^{2}=0$, and thus $m x=0, m=0$.

Suppose now that $x y z^{p}=x y^{p} x^{p-1}=0$ in $\hat{G}$. Then the algebra $\hat{G}_{3}$ is nilpotent $\hat{G}_{3}^{3 p-2}=0=G_{3}^{3 p-2}$. Hence $G_{3}$ has these identities. Since $x y_{z} p_{z}=$ $z y^{p} x+x z y^{p}$ in $\hat{G}_{3}$ (see $\left[1\right.$, p. 58]), we have in $G_{3}$ :

$$
J(x, y, z)+\beta x y^{p} z+\gamma x z^{p} y+\delta y x^{p} z=0, \quad \beta, \gamma, \delta \in \mathrm{F}_{q} .
$$

If, for example, $\beta \neq 0$, the linearization of (5) by $y$ gives us $x_{1} \cdots x_{p+2}=0$ (see [1, Lemma 2, p. 58]); thus $J(x, y, z)=0$, that is, $G$ is a Lie algebra.

Similarly, $(x y)(z t)+\beta x y^{p} z t+\cdots=0$ implies $(x y)(z t)=0$.

Suppose now that $x_{1} \cdots x_{p+3}=0$ in $\hat{G}$ and, hence, in $G$. Then $G$ has all identities of $\hat{G}$ of degree $p+2$. By Proposition $1(x y)(z t) \in G_{4}^{p+3}=0$. Similarly $J(x, y, z) \in G_{3}^{p+2}$ and therefore

$$
J(x, y, z)=\left(\beta x y^{p} z+\cdots\right)+\left(\gamma x y z^{p}+\cdots\right) .
$$

If $\beta \neq 0$ then, again by $\left[1\right.$, Lemma 2, p. 58], we have $x y^{p} z=0$. So, we can suppose that

$$
J(x, y, z)=\beta x y z^{p}+\gamma y z x^{p}+\delta z x y^{p}, \quad \beta, \gamma, \delta \in \mathbf{F}_{q} .
$$

If $q>p$ then, by Proposition $1, J(x, y, z)=0$, and in this case the theorem is proved.

Suppose now that $p=2, x_{1} \cdots x_{5}=0$ and $y x^{2} y=0$. As it has been noticed in [1, p. 59], $y x^{2} y=0$ implies $x y z^{2}=0$ and thus $J(x, y, z)=0$ by (5'). If $y x z t+$ 
$z x y t+t x y z=0$ put $t=z$ and obtain $y x z^{2}=0$ and then $J(x, y, z)=0$.

If $p \geqslant 3$ and $x y^{p+1}=0$, then as it has been noticed in [1, p. 58], we have $x y z^{p}=z x y^{p}$ and so $J(x, y, z)=\beta x y z^{p}$.

If $p \geqslant 3$ and $x y z^{p}+y z x^{p}+z x y^{p}=0$, then we can assume that in (5') $\delta=0$. If $\beta \neq 0$ in $\left(5^{\prime}\right)$ put $y=z$. We obtain $x y^{p+1}=0$ and as above $x y z^{p}=$ $z x y^{p}=y z x^{p}$, which implies $J(x, y, z)=0$.

Finally suppose that $p=3, x y^{4} \neq 0$, put $y=z$ in (5'). We have $\beta x y^{4}-$ $\delta x y^{4}=0$ and, therefore, $\beta=\delta$. Similarly $\beta=\gamma=\delta$, i.e.

$$
J(x, y, z)=\beta\left(x y z^{p}+y z x^{p}+z x y^{p}\right) .
$$

To finish the proof we need to show that if $p \geqslant 3$,

$$
x^{2}=(x y)(z t)=x y^{p+1}=J(x, y, z)-\beta x y z^{p}=x_{1} \cdots x_{p+3}=0
$$

or $p=3$,

$$
x^{2}=(x y)(z t)=x y z t=J(x, y, z)-\beta\left(x y z^{3}+z x y^{3}+y z x^{3}\right)=0,
$$

then $V$ has a chain of subvarieties. In the first case if $f=0$ is any element in $G$, then modulo verbal ideal generated by $x y z^{p}$ it is (see $[1$, p. 61]) equivalent to $x_{1} \cdots x_{n}=0, n<p+3$, or $\Sigma_{i} f_{i, p}=0$, where $f_{i, p}=x_{i} x_{1} \cdots \hat{x}_{i} \cdots x_{p}$, and each of them implies $x y z^{p}=0$. So subvarieties of $V$ form a chain. The second case is analogous. This completes the proof.

Suppose now that $p=2$. The algebra $\hat{G}$ satisfies one of the sets of identities from [1, Theorem 8]. The cases of commutative and associative algebras, metabelian Lie algebras have been already considered. So we can suppose that $\hat{G}$ satisfies identities (3)-(7) from Theorem 8. In the case (3) we have $\hat{G}^{4}=G^{4}$ $=0$ and since $\hat{G}$ was defined by identities of degree three, $G$ has the same identities. If $G^{2} \neq 0$, then $\alpha G_{1}=\mathfrak{m} G_{1} \subseteq G_{1}^{2}$, i.e. $\alpha x=\beta x^{2}+\gamma x^{2} x, \alpha \in \mathfrak{m}, \beta, \gamma \in k$. Multiplying twice by $x$ we have $\alpha x^{2}=\beta x^{2} x, \alpha x^{2} x=0$. So $k x^{2} x=\mathrm{F}_{q} x^{2} x$. If $\gamma \in \mathrm{F}_{q}^{*}$ then by [1, p. 76], the linearization of $x^{2} x$ is equivalent to $x^{2} y=0$. Thus, without loss of generality we can suppose that $\gamma=0$. So $\alpha x=\beta x^{2}$. If $\beta \in k^{*}=k \backslash \mathrm{m}$, then

$$
0=(x+y)^{2}-\alpha \beta^{-1}(x+y)-x^{2}+\alpha \beta^{-1} x-y^{2}+\alpha \bar{\beta}^{-1} y=x y+y x
$$

and, hence, $x y z=0$ in $G$, i.e. $G$ is commutative and associative. If $\beta=\alpha \gamma, \gamma \in$ $k$, then $\alpha x^{2}=\alpha \gamma x^{2} x=0$ and therefore $\alpha x=\alpha \gamma x^{2}=0$.

Similarly, if $\hat{G}$ satisfies identities of type (6)-(7) from Theorem 8 , then $\hat{G}=G$.

Suppose now that $\hat{G}$ is of type (4), that is $J(x, y, z)=0, x(y z)=\gamma x y z+$ $\delta y x z, y z x=\beta^{2} x y z+\left(\beta^{2}+\beta+1\right) y x z, \hat{G}^{5}=G^{5}=0$. As was mentioned in 
$\left[1\right.$, p. 77], in this case every $f=f(x, y, z) \in G^{4}$ is a linear combination of $x^{2} y z$, $y^{2} z x, z^{2} y x$ and a linearization of $x^{2} y z$ is equivalent to $x y z t=0$. Thus, if $J(x, y, z)+\beta x^{2} y z+\gamma y^{2} z x+\delta z^{2} x y=0$ in $G$ and if $\beta \neq 0$, then the linearization of this identity gives $x y z t=0$ and, hence, $J(x, y, z)=0$ in $G$. Similarly we consider the other identities and a case (5).

Finally, since $J(x, x, x)=J_{1}(x, x, x)=x^{2} x=x x^{2}=0$, then if $G^{2} \neq 0$, $\alpha G_{1} \subseteq G_{1}^{2}$, that is $\alpha x=\beta^{\prime} x^{2}$ and as in the preceding case if $G^{3} \neq 0$ then $\alpha=0$.

Eventually we have proved

THEOREM 4. Let $p=2$ and $G$ is not commutative, associative or metabelian Lie algebra. Then $\mathrm{m}=0, \hat{G}=G$ satisfies one of the following sets of identities (numeration from [1, Theorem 8]):

(iii) $x y z t=0$ and either $x(y z)=(\alpha+\beta) x y z+\beta y x z, \alpha \in F_{q} \backslash 1, x y z=y z x$, or $x y z=\alpha z(x y+y x), x(y z)=y(z x)$,

(iv) $y x z t u=0, J(x, y, z)=0, y z x=\beta^{2} x y z+\left(\beta^{2}+\beta+1\right) y x z, x(y z)=$ $\delta^{\prime} y x z+\gamma^{\prime} x y z, \beta, \delta^{\prime}, \gamma^{\prime} \in F_{q}$,

(v) $x y z t u=0, J_{1}(x, y, z)=0, x(z y)=\delta^{2} z(y x)+\left(\delta^{2}+\delta+1\right) z(x y)$, $x y z=\alpha^{\prime} z(x y)+\beta^{\prime} z(y x)$,

(vi) $J(x, y, z)=0, x(y z)=\delta y z x, \delta \neq 1, x y z=y x z$,

(vii) $J(x, y, z)=x y z=0, x(y z)=x(z y)$.

Here $J(x, y, z)=x y z+y z x+z x y, J_{1}(x, y, z)=x(y z)+y(z x)+z(x y)$.

Assume now that $p>2$.

THEOREM 5. If $\hat{G}, G^{2} \neq 0$, satisfies identities $x y-y x=x^{3}=(x y)(z t)=$ 0 , then $\mathrm{m}=0, \hat{G}=G$ and $V$ has a chain of subvarieties.

Proof. As in [1] we have $x y z t=-x y t z, x y z^{2}=0$ and hence $G_{3}^{5}=0$. Thus, by Proposition $1, x^{3}=(x y)(z t)=0$. Moreover, if $x$ appears at least three times in a monomial $f$, then $f=0$. Thus, $G$ is commutative. Finally, $m G \subseteq G^{2}$ implies, by Proposition $1, \mathfrak{m}=0$.

THEOREM 6. If $\hat{G}$ is a 3-Engel Lie algebra, $p>2, G^{2} \neq 0$, then $\hat{G}=G$, $\mathrm{m}=0$ and $V$ has a chain of subvarieties.

Proof. Since $x^{2}=0$ in $\hat{G}, \hat{G}_{1}^{2}=G_{1}^{2}=0$ and $m G_{1} \subseteq G_{1}^{2}=0$, i.e. $m=0$. Note that by Theorem 7 [1] if $p=3$ then $\hat{G}^{5}=G^{5}=0$, if $p>3$ then $\hat{G}_{3}^{6}=$ $G_{3}^{6}=0$. Thus by Proposition 1 we have $J(x, y, z), x y^{3} \in G_{3}^{7}=0$.

THEOREM 7. If $p=3$ and $\hat{G}$ satisfies one of the following sets of identities (numeration from [1, Theorem 4$]), G^{2} \neq 0$,

(v) $x^{2}=x y z t u=0, x y z t=(\alpha+\beta)(t z)(y x)-\alpha(t y)(z x)+\alpha(t x)(z y)$, where either $\alpha \neq 0$ or $\beta \neq 0$, 
(vi) $x^{2}=x y z t u=0$ and either $x y t z=(\gamma-1) x y z t-\gamma(\gamma+1) \gamma(x, y, z) t$, $t x y z=\gamma x y z t+y z x t-\gamma^{2} J(x, y, z) t, \gamma \in \mathrm{F}_{q}$, or $x y z t=x y t z+y z t x+z x t y$,

(vii) $x^{2}=x y z t u=0, x y t z+x y z t=t x y z+y z x t=0$, then $\mathrm{m}=0, \hat{G}=G$ and $V$ has $a$ chain of subvarieties.

Proof is obvious, since $x^{2}=0$ in $\hat{G}$ implies $m=x^{2}=0$ in $G$. Also $\hat{G}^{5}=$ $G^{5}=0$ and $\hat{G}=G$ since $G$ is defined by identities of degree four.

THEOREM 8. If $p>3$ and $\hat{G}, G^{2} \neq 0$ has either identities $x^{2}=x y z+$ $x(y z)=x y z t=0$ or $x y-y x=x^{3}=x y z t-x y t z=x y z t u=0$, then $\mathfrak{m}=0, \hat{G}=G$ and in these cases $V$ has $a$ chain of subvarieties.

Proof. In the first case $x^{2}=0$ implies as usual $m=0$ and since $x y z t=0$ by Proposition 1, $\hat{G}=G$. In the second case $m G_{1} \subseteq G_{1}^{2}$ and $x^{3}=0, p>3$, imply by Proposition $1, \mathfrak{m}=0$. Now $x y-y x \in \hat{G}_{2}^{6}=G_{2}^{6}=0$. Thus, $\hat{G}=G$.

3. Power-associative algebras. Let $k$ be a Noetherian commutative, associative ring, $V$ variety of power-associative algebras, $F=G_{1}$ a $V$-free algebra with one generator $x$. Then $F$ is a Noetherian commutative associative ring. Let $J$ be the Jacobson radical of $F$. If $J=F$ then by results from [7, p. 12], $\wedge_{n} F^{n}=$ 0 , so we can apply to var $F$ Theorem 2 and obtain

THEOREM 9. If $J=F \neq 0$, then $x^{n}=0,2 \leqslant n \leqslant p+1, k$ has a unique maximal ideal $m$ and if $\mathfrak{m} \neq 0$, then either $k / \mathfrak{m}=F_{p}, \mathfrak{m}=F_{p} \alpha, \alpha x=x^{p}, x^{p+1}=$ $\alpha^{2}=\alpha x^{2}=0$ or $x^{2}=0$.

Suppose now that $F \neq J$. Then $F / J$ is a subdirect product of fields $K_{j}$, $F / J \subseteq \Pi K_{j}$. Each $K_{j}$ from this decomposition is a $k$-algebra, that is we have ring homomorphisms $f_{j}: k \rightarrow K_{j}, \operatorname{Ker} f_{j}=\mathfrak{p}_{j}$. The ideal $\mathfrak{p}_{j}$ is prime and $k_{j}=$ $k / \mathfrak{p}_{j} \in \operatorname{var} K_{j} \subseteq V$. If $L \triangleleft k_{j}$, then $L=$ Ann var $k_{j} / L$ and, therefore, ideals of $k_{j}$ form a chain, that is $k_{j}$ is a valuation ring (see [7]) with unique maximal principal ideal $\left(\delta_{j}\right)$ and every ideal of $k_{j}$ is equal to some $\left(\delta_{j}^{t}\right), t \geqslant 0$.

Now $K_{j}$ is generated by $k_{j}$ and the image of $x$, which is different from zero, since $F / J \neq 0$. By (3), $\left(3^{\prime}\right)$ we have in $K_{j}: \delta_{j}^{(d(d-1)+2) / 2} x^{2}=0$, and since $K_{j}$ is a field, $\delta_{j}=0$. Thus, $k_{j}$ is a field as well. For distinct $i, j$ we have, for example,

$$
p_{i}=\text { Ann var } k_{i} \subseteq \text { Ann var } k_{j}=p_{j}
$$

and since $k_{i}, k_{j}$ are fields, $\mathfrak{p}_{i}=\mathfrak{p}_{j}=\mathfrak{m}$. Note that var $J / J^{n} \not \supset K_{j}$, hence, var $K_{j}$ $\ni J / J^{n}$ and, therefore,

$$
\mathfrak{m}=\text { Ann } \operatorname{var} K_{j}=\text { Ann } \operatorname{var} F / J \subseteq \text { Ann } \operatorname{var} J / J^{n}
$$

for all $n>1$. But $m$ is a maximal ideal. Thus, either $m=A n n$ var $J / J^{n}$ and 
$m J \subseteq \bigwedge_{n} J^{n}=0$ (see $\left[7\right.$, p. 12]) or $J=J^{n}=\bigwedge_{n} J^{n}=0$. In both cases $m^{2} F \subseteq$ $\mathrm{m} J=0$ and therefore $m$ is a unique maximal ideal.

Suppose now, that $k / m=F_{q}$. Then each $K_{j}$ is a $F_{q}$-algebra, var $F_{q} \subseteq V$ and hence $\operatorname{var} \mathbf{F}_{q} \supseteq \operatorname{var} J / J^{2}$. But in $\operatorname{var} \mathbf{F}_{q}$ each finite $\mathbf{F}_{q}$-algebra is a direct product of $F_{q}$ (see, for example, [8]). So, $J / J^{2} \in$ var $F_{q}$ implies $J=J^{2}$ and therefore $J=0, m F \subseteq J=0$. Eventually, we have

THEOREM 10. If $J \neq F$, then $k$ has a unique maximal ideal $m, m^{2}=0$. If $k / m=F_{q}$, then $J=0, m=0, k=F_{q}$.

Since the case $|\mathrm{k} / \mathrm{m}|=\infty$ has been reduced by Theorem 1 to the results of [1] we assume throughout this paper, by Theorems 9 and $10, k / m=F_{q}$, and if $J=F$, then $m=\mathbf{F}_{q} \alpha, \alpha^{2}=0$, if $J \neq F$, then $J=m=0$.

4. Alternative algebras. We need

Proposition 5. Let $A$ be a right alternative $k$-algebra (see [9]). If $A$ is anticommutative, then $y x z+y(x z)=0$ and if char $\mathrm{F}_{q} \neq 2$, then $x y z t=0$. If $A$ is commutative (and, hence, alternative), $p=\operatorname{char} \mathrm{F}_{q} \neq 3$, then $A$ is associative. If $A$ is commutative, $p=3$, then $A$ is alternative and $J(x, y, z)=0$. Conversely, if $A$ is commutative, $p=3$ and $J(x, y, z)=0$, then $A$ is alternative.

Proof. If $A$ is anticommutative and $(x, y, z)+(x, z, y)=0$, then

$$
\begin{aligned}
x y z & =(x, y, z)+x(y z)=-(x, z, y)-y z x=-x z y+x(z y)-y z x \\
& =z x y-z y x-y z x=z x y=y z x=-x(y z) .
\end{aligned}
$$

If $p>2$, then

$$
x y z t=-(x(y z)) t=x((y z) t)=-x(y(z t))=(x y)(z t)=-x y z t,
$$

i.e. $x y z t=0$.

If $A$ is commutative, then

$$
x y z=(x, y, z)+x(y z)=-(y, x, z)+y z x=-x y z+x z y+y z x,
$$

and, hence,

$$
2 x y z=x z y+y z x, \quad 2 y z x=y x z+z x y .
$$

From (6) $3 x y z=3 x z y$ and since $p \neq 3, x y z=x z y=y x z=y z x=x(y z)$. If $p=3$, then in (6) $J(x, y, z)=0$.

Conversely, if $J(x, y, z)=0, p=3$, then

$$
x^{2} y-x(x y)=x^{2} y-x y x=x^{2} y+2 x y x=J(x, y, x)=0 .
$$

Similarly, $y x^{2}=y x x$. 
Let $V$ be a variety of alternative $k$-algebras. Then $F=G_{1}$ satisfies conditions of Theorems 9 and 10. If $J=F$ and, therefore, $x^{p+1}=0$, then by a theorem of Shirshov-McCrimmon (see [10]), $V$ is locally nilpotent and $V$-free algebras have property (2). Applying Theorems 2-8 we have

THEOREM 11. Let $V$ be a variety of alternative $k$-algebras with $x^{p+1}=0$. Then $V$ is one of varieties:

(i) any variety of associative commutative algebras from Theorem 2;

(ii) $p \neq 2, x y+y x=x y z+x(y z)=x y z t=0, m=0$;

(iii) $p=3, x y=y x, x^{3}=(x y)(z t)=0, m=0$;

(iv) $p=2, x y z t=0$ and either $x(y z)=\alpha x y z, \alpha \neq 1, x y z=y z x$, or $x(y z)=$ $y(z x)=y(x z), x y z=0$; in both cases $\mathrm{m}=0$;

(v) $p=2,(x, y, z)=J(x, y, z)=x y z t=0, m=0$;

(vi) $p=2,(x, y, z)=J(x, y, z)=y z x+\beta^{2} x y z+\left(\beta^{2}+\beta+1\right) y x z=x y z t$ $=0, \beta \neq 1, m=0$.

Note. This theorem is valid when $k$ is an infinite field, char $k>0$. If $k$ is a field of characteristic zero, then $V$ is either of type (ii) or any variety of commutative and associative algebras.

Proof. By Proposition 5 we can suppose that $V$ is not anticommutative and if $G$ is commutative, but not associative, then $p=3, J(x, y, z)=0$ and in this case, by Theorems $5-6, V$ has identities $x^{3}=(x y)(z t)$. Note that linearization of $x^{3}=0$ gives us $J(x, y, z)$, so we have (iii).

Suppose now that $p=2$ and $V$ of types (iii)-(vii) from Theorem 4. In case (iii), $x^{3}=x^{2} x=(\alpha+\beta) x^{2} x+\beta x^{2} x=\alpha x^{2} x$, where $\alpha \neq 1$. So, $x^{3}=0$. But, by results from [1, p. 79], the linearization of $x^{3}=0$ implies $x^{2} y=0$ and, hence, $x(y z)=\alpha x y z, \alpha \neq 1$. The dual case in (iii) is similar.

Now if $G$ is of types (iv)-(vii) from Theorem 4 , then $J(x, y, z)=$ $J_{1}(x, y, z)=0$ and by alternativity,

$$
0=x y z+x(y z)+y z x+y(z x)=z x y+z(y x),
$$

i.e. $V$ is associative. In cases (iv) and (v) we have

$$
J(x, y, z)=y z x+\beta^{2} x y z+\left(\beta^{2}+\beta+1\right)=0
$$

or

$$
x z x=\beta^{2} x^{2} z+\left(\beta^{2}+\beta+1\right) x^{2} z=(\beta+1) x^{2} z,
$$

and by $J(x, x, z)=0$ we have $z x^{2}=\beta x^{2} z$. As we have noticed in $[1, \mathrm{p} .77]$, in this variety $x y z t=y x t z=z t x y$. Thus, $x^{2} z t=z t x^{2}=\beta x^{2} z t$. So, if $\beta \neq 1$, then $x^{2} z t=0$ and $x y z t=y x z t$, i.e. since $\mathrm{F}_{q} S_{4} x y z t$ is the irreducible two-dimensional $\mathrm{F}_{q} S_{4}$-module, $x y z t=0$. 
Now consider case (vi) from Theorem 4. $V$ is associative and $x y z=y x z$, $x y z=\delta y z x, \delta \neq 1$. Then $x y z=\delta y z x=\delta z y x=\delta^{2} y x z=\delta^{2} x y z$. Since $\delta \neq 1$ this implies $x y z=0$. Similarly, in (vii) we have $x y z=0$. So, in both cases $V$ is of type (v). By Proposition 5 this completes the proof.

THEOREM 12. If in $F=G_{1}$ we have $J(F)=0, k=\mathbf{F}_{q}$, then $V=\operatorname{var} \mathbf{F}_{q^{r}}$, where $r=1$, or $r$ is a prime-power. In this case subvarieties of $V$ form a chain and $V$ is defined by identity $X=X^{q^{r}}$.

Proof. If $x \in F$ is not algebraic, then $F=(X) \triangleleft \mathrm{F}_{q}[X]$ and, thus, $F$ contains two verbal ideals $\left(X^{2}\right),\left(X-X^{q}\right)$, which do not contain one another. Thus, $x$ is algebraic, $\operatorname{dim}_{k} F<\infty$ and since $J(F)=0, F=\bigoplus_{i=1}^{n} \mathbf{F}_{q} d_{i}$ and therefore, $\operatorname{var} F=\operatorname{var} \mathbf{F}_{q^{r}}$ for some $\mathbf{F}_{q^{r}}$ from this decomposition. By Jacobson's theorem (see [6], [11]) two-generated algebras in $V$ are commutative and hence, $V$ is associative (see [12, Lemma 3]), since $V$ has an identity $X=X^{q^{r}}$.

Lemma 1. If $z \in Z, z>1$, then $\left(z^{s}-1\right) \mid\left(z^{t}-1\right)$ iff s|t.

Proof. Consider the ring $R=Z /\left(z^{s}-1\right)$. The element $z \in R^{*}$ has order s. So $z^{t}=1$ iff $s \mid t$.

Lemma 2. The field $\mathrm{F}_{q^{s}}$ satisfies the identity $X=X^{q^{t}}$ iff $s \mid t$.

Proof. Take $\beta$ to be a generator of $\mathbf{F}_{q^{s}}^{*}$. Then $\beta$ has order $q^{s}-1$. Thus, $\beta^{q^{t}-1}=1$ iff $\left(q^{s}-1\right) \mid\left(q^{t}-1\right)$ and iff $s \mid t$ by Lemma 1 .

Now $V$ has the identity $X=X^{q}$ and by Lemma 2 and Jacobson's theorem each algebra $A \in V$ is a subdirect product of subfields of $\mathrm{F}_{q^{r}}$. So $V=\operatorname{var} \mathbf{F}_{q^{r}}$ and $V$ has a chain of subvarieties iff $r=1$ or $r$ is a prime power.

5. $(\gamma, \delta)$-algebras. In this part we make some remarks concerning $(\gamma, \delta)$-algebras. Recall that algebra $A$ over a field $k$ is a $(\gamma, \delta)$-algebra iff

$$
\begin{aligned}
(x, y, z)+\gamma(y, x, z)+\delta(z, x, y) & =0, \\
(x, y, z)-\gamma(x, z, y)+(1-\delta)(y, z, x) & =0
\end{aligned}
$$

where $\gamma, \delta \in k$ and $\gamma^{2}-\delta^{2}+\delta-1=0$.

As it has been shown in $[13$, p. 518], if char $k \neq 2,3,5$, then $A$ is powerassociative. Moreover, if char $k \neq 2,3$ and $A$ has no nilpotent elements, then $A$ is associative [13, Theorem 1, p. 516]. In the special case $\gamma=-1, \delta=1$, if char $k \neq 2$, then $A$ is power-associative, and if char $k \neq 2,3$ and $x^{n}=0$ for all $x \in A$, then $A$ is locally nilpotent (see [14]), and, hence, $\operatorname{var} A$ has property (2). Thus, we have

THEOREM 13. Let $V$ be a variety of $(\gamma, \delta)$-algebras over a field $\mathbf{F}_{q}$, where $p=\operatorname{char} \mathbf{F}_{q}>5$ for arbitrary $(\gamma, \delta)$ and $p>3$ for $\gamma=-1, \delta=1$. If $V$ is not a 
variety of nilalgebras, then by Theorem $12, V=\operatorname{var} \mathrm{F}_{q}$, where $r=1$ or $r$ is a prime-power. If $\gamma=-1, \delta=1, p>3$ and $V$ is a variety of nilalgebras, then $V$ is one of the following varieties:

(i) variety of commutative, associative algebras from Theorem 2,

(ii) $p>2, x y+y x=x y z+x(y z)=x y z t=0$,

(iii) $p=3, x y=y x, x^{3}=(x y)(z t)=0$.

The proof by Proposition 5 follows from Theorems 5-8 since $(-1,1)$-algebras are right alternative.

Note that if $k$ is an infinite field, char $k \neq 2,3$ then (i)-(ii) are the only varieties of $(-1,1)$-algebras with a chain of subvarieties, since in this case they are homogeneous.

6. Jordan algebras. Throughout this part, $V$ is a variety of Jordan algebras, $k$ from Theorems 9 and 10, and as usual in this case, $p=\operatorname{char} \mathrm{F}_{q}>2$.

Assume first that $F=G_{1}$ satisfies conditions of Theorem 10. Consider the Jordan $\mathrm{F}_{q}$-algebra $A_{\delta}$ which is a subalgebra of the matrix algebra $\operatorname{Mat}\left(2, \mathrm{~F}_{q}\right)^{+}$, consisting of all matrices

$$
T=\left(\begin{array}{ll}
\alpha & \beta \delta \\
\beta & \gamma
\end{array}\right)
$$

where $\alpha, \beta, \delta, \gamma \in \mathrm{F}_{q^{s}}, \delta$ is fixed, $-\delta \notin \mathrm{F}_{q^{s}}^{2}$. So, $\operatorname{dim}_{\mathrm{F}_{q^{s}}{ }{ }_{\delta}}=3$.

Proposition 6. Algebras $A_{\delta}$ for distinct $\delta$ are isomorphic.

Proof. Define the map $f: A_{\delta} \rightarrow A_{\delta \gamma^{2}}, \delta, \gamma \in \mathrm{F}_{q}^{*}$, by

$$
\left(\begin{array}{ll}
\alpha_{1} & \beta \delta \\
\beta & \alpha_{2}
\end{array}\right)^{f}=\left(\begin{array}{cc}
\alpha_{1} & \beta \delta \gamma \\
\beta \gamma^{-1} & \alpha_{2}
\end{array}\right)
$$

An easy calculation shows that $f$ is a homomorphism, and, therefore, an isomorphism.

Proposition 7. If $\beta \neq 0$ in (7), then eigenvalues of $T$ are distinct.

Proof. The characteristic polynomial of $T$ is $X^{2}-X(\alpha+\gamma)+(\alpha \gamma-$ $\beta^{2} \delta$ ). If its roots coincide, then

$$
0=(\alpha+\gamma)^{2}-4 \alpha \gamma+4 \beta^{2} \delta=(\alpha-\gamma)^{2}+(2 \beta)^{2} \delta
$$

or $-\delta=\left[(2 \beta)^{-1}(\alpha-\gamma)\right]^{2}$, which is impossible. 
Note that eigenvalues of $T$ belong to $\mathrm{F}_{q^{2 s}}$.

Proposition 8. There exists $T \in A_{\delta}$ such that the eigenvalues of $T$ belong to $\mathbf{F}_{q^{2 s}} \backslash \mathbf{F}_{q^{s}}$.

Proof. Suppose that for all $T \in A_{\delta}$ the eigenvalues belong to $\mathrm{F}_{q^{s .}}$. Then for all $\alpha, \beta, \gamma \in \mathbf{F}_{q^{s}}$,

$$
(\alpha+\gamma)^{2}-4 \alpha \gamma+4 \delta \beta^{2}=(\alpha-\gamma)^{2}+(2 \beta)^{2} \delta \in \mathrm{F}^{2},
$$

that is for all $x, y \in \mathbf{F}_{q^{s}}$ there exists $z \in \mathbf{F}_{q^{s}}$ such that $x^{2}+y^{2} \delta=z^{2}$. Hence, $0+1 \cdot \delta=u^{2}$ and $x^{2^{q}}+y^{2} \delta=x^{2}+(u y)^{q^{2}}=z^{2}$. Since $\delta \neq 0, u \neq 0$ and for all $x, y \in \mathrm{F}_{q^{s}}$ there exists $z \in \mathrm{F}_{q^{s}}$ such that $x^{2}+y^{2}=z^{2}$. Thus, $\mathbf{F}_{q^{s}}^{2}$ is a subgroup of $\mathrm{F}_{q^{s}}^{q^{s}}$. Since $\delta \in \mathrm{F}_{q^{s}}^{2},-\delta \in \mathrm{F}_{q^{s}}^{2}$. However, this contradicts our assumption.

Proposition 9. The algebra $A_{\delta}$ satisfies the identity $X=X^{2 s}$, not the identity $X=X^{q^{s}}$.

Proof. By Proposition 7 the matrix $T$ from equation (7) is conjugate in $\operatorname{Mat}\left(2, \mathbf{F}_{q}{ }_{q s}\right)$ to the matrix

$$
T_{1}=\left(\begin{array}{cc}
\alpha_{1} & 0 \\
0 & \alpha_{2}
\end{array}\right), \quad \alpha_{1}, \alpha_{2} \in F_{q^{2 s},}
$$

and, therefore, $T_{1}^{q^{2 s}}=T_{1}$. The affirmation follows now from Propositions 7 and 8.

THEOREM 14. Let $V$ be a variety of Jordan $\mathbf{F}_{q}$-algebras, $p=\operatorname{char} \mathbf{F}_{q}>2$, and $X=X^{q}$ in $V$, where $r=1$ or $r$ is a prime-power. Then either $V=\operatorname{var} F_{q^{r}}$ or $r=2, V=\operatorname{var} A_{\delta}$, where $A_{\delta}$ is the $\mathrm{F}_{q}$-algebra, defined above (with $s=1$ ).

Proof. Let $B \in V$. By Theorems 15.11, 15.4 from [6] and Proposition 6, $B$ is a subdirect product of fields $\mathrm{F}_{q^{t}}, t \mid r$, and algebras $A_{\delta}$ over some fields $\mathrm{F}_{q^{s}}$ By Proposition 6 we assume $\delta \in \mathrm{F}_{q}^{q^{t}}$. By Proposition 9 since $\mathrm{F}_{q^{s}} \in V$ and $s \mid r$ we have $\mathbf{F}_{q}[\alpha] \in V$ for some $\alpha \in \mathbf{F}_{q^{2 s}} \backslash \mathbf{F}_{q^{s}}$, i.e. by Lemma 2 from Theorem 12 we have $2 \mid r$. Thus, $r=2^{n}$ and $F_{q}{ }_{q s}=F_{q}[\alpha] \in V$.

LEMma 1. If $A_{\delta} \in V$, then $s=1$.

Proof. Let $A_{\delta}^{\prime}$ be a $\mathrm{F}_{q}$-subalgebra of $A_{\delta}$ with $\alpha, \beta, \gamma \in \mathrm{F}_{q}$ in (7). Note that by our assumption $\delta \in \mathrm{F}_{q}$. If $\operatorname{var} \mathrm{F}_{q^{2 s}} \ni A_{\delta}^{\prime}$, then as we have already noticed at the end of $\S 4$ of this paper, $A_{\delta}^{\prime}$ is a subdirect product of fields $\mathbf{F}_{q^{t}}$. But $A_{\delta}^{\prime}$ is a simple algebra (see [6, Proposition 15.4]), so $A_{\delta}^{\prime} \simeq \mathrm{F}_{q}$, which is impossible since $A_{\delta}^{\prime}$ has nontrivial idempotents. Thus, $F_{q^{2 s}} \in \operatorname{var}^{q} A_{\delta}^{\prime}$ and by 
Proposition 9, Lemma 2 from Theorem 12, we have 2s/2, i.e. $s=1, A_{\delta}^{\prime}=A_{\delta}$.

Finally, every algebra $B \in \operatorname{var} A_{\delta}$ over $\mathrm{F}_{q}$ satisfies the identity $X=X q^{2}$ and by results of [6], $B$ is a subdirect product of some copies of $F_{q}, F_{q^{2}}$ and $A_{\delta}$ (see also Proposition 6). Hence there is the only chain

$$
0 \subset \operatorname{var} \mathrm{F}_{q} \subset \operatorname{var} \mathrm{F}_{q^{2}} \subset \operatorname{var} A_{\delta}=V
$$

of subvarieties. This completes the proof.

Now suppose that the $V$-free algebra $G_{1}=F$ enjoys conditions of Theorem 9.

THEOREM 15. If $F$ enjoys conditions of Theorem $9, G^{2} \neq 0$, then $V$ is one of the varieties:

(i) commutative and associative algebras from Theorem $2, p \neq 2$,

(ii) $p \neq 2, x y=y x, x^{3}=(x y)(z t)=m=0$,

(iii) $p \neq 2,3, x y=y x, x^{3}=x y z t-x y t z=x y z t u=m=0$.

Proof. Since $V$ is a variety of nilalgebras, $x^{p+1}=0$, by results of Shirshov [15] special algebras in $V$ are locally nilpotent. Thus $U=\operatorname{var} G_{2}$ has property (2) and therefore $U$ is a commutative variety from Theorems 2,5 and 8. If $U$ is associative, then $V$ is a variety of alternative algebras and by Theorem 11, $V$ is of types (i) or (ii), since $V$ is commutative.

Suppose now, that in $U$ we have $x y-y x=x^{3}=(x y)(z t)=0$. Then in $V$ we have $x y-y x=x^{3}=x^{2} \cdot y^{2}=0$. Since $V$ is commutative, linearization of $x^{2} \cdot y^{2}=0$ gives us

$$
0=(x+y)^{2} \cdot z^{2}-x^{2} \cdot z^{2}-y^{2} \cdot z^{2}=2(x y) \cdot z^{2}
$$

and $(x y) \cdot z^{2}=0$. Similarly, $(x y)(z t)=0$, i.e. $V$ is of type (ii).

Suppose finally that $U$ has identities

$$
x y=y x, \quad x^{3}=0, \quad x y z t=x y t z, \quad x y z t u=0, \quad p \neq 2,3 .
$$

The linearization of $x^{3}=0$ gives us $J(x, y, z)=0$, i.e. in $V$ we have $x y=y x$, $x^{3}=J(x, y, z)=0$. Moreover, in $U$, and, hence, in $V$ we have

$$
\begin{gathered}
x^{2} y x=x^{3} y=0, \quad y x x x=x y x x=-1 / 2 x^{3} y=0, \\
J(x, x, y)=x^{2} y+2 x y x=0, \quad x y x=y x x .
\end{gathered}
$$

By linearization of $x^{2} y x=0$ we obtain

$$
x^{2} y z+2 z x y x=0 .
$$

Also, by linearization of $x y x y-x y y x=0$ by $x$ and $y$ we have

$$
x y x z+x z x y+y z x x=0, \quad x y z z+x z z y+y z z x=0 .
$$


LemMA. Let $G_{3}$ be a $V$-free algebra with free generators $x=x_{1}, y=x_{2}$, $z=x_{3}$, and $M \in G_{3}$ a monomial

$$
M=t_{1} \cdots t_{n}, \quad n>2, \quad t_{t}=x_{1}, x_{2}, x_{3} .
$$

If $x_{m}$ appears $j_{m}$ times in $M$, then

$$
M=\beta x_{i_{1}}^{i_{i_{1}}} \underbrace{x_{i_{2}} x_{i_{3}} x_{i_{3}}}_{i_{i_{2}} \cdots x_{i_{2}}} \underbrace{}_{i_{i_{3}}}, \quad \beta \in k^{*},
$$

$\left(i_{1}, i_{2}, i_{3}\right)$ is a permutation of $(1,2,3)$.

COROLlaRY. If $x$ (or $y, z)$ appears in $M$ at least three times, then by (8), $M=0$.

Proof of Lemma. The case $n=3$ is trivial by (8). Suppose that $n>3$, and for $n-1$ the Lemma was proved. By induction, if $M \neq 0$ we can assume that

$$
t_{1} \cdots t_{n-1}=\underbrace{x \cdots x}_{j_{1}} \underbrace{\cdots \cdots y}_{j_{2}} \underbrace{z \cdots z}_{j_{3}}, \quad 0 \leqslant j_{i}<3, j_{1}+j_{2}+j_{3}>2 .
$$

The case $t_{n}=z$ is trivial. So, $t_{n}=x, y$. Note that by (8) and $x y x y=x y y x$,

$$
\underbrace{x \cdots x}_{j_{1}} \underbrace{y \cdots y}_{i_{2}}=\gamma \underbrace{y \cdots y}_{j_{2}} \underbrace{x \cdots x}_{i_{1}}, \quad \gamma \in k^{*} .
$$

So without loss of generality we can suppose that $t_{n}=y, j_{2}>0$. If $j_{1}=2$, then put $u=x^{2}$ and applying an induction and identity

$$
(y z) \cdot x^{2}=x^{2}(y z)=-x(y z) x-y z x x=-2 y z x x,
$$

we prove our Lemma.

So $j_{1}=1$ (the case $j_{1}=0$ is trivial as we have already noticed),

$$
M=x \underbrace{\cdots}_{j_{2}} \overbrace{j_{3}}^{z \cdots z y} .
$$

If $j_{3}=2$, then by $\left(8^{\prime \prime \prime}\right)$ we have

$$
M=-1 / 2(x y \cdot \cdots y) \cdot z^{2} \cdot y \text {. }
$$

So applying induction to $M^{\prime}=x y \cdots y z y$ and using $\left(8^{\prime \prime \prime}\right)$ we prove the Lemma. Suppose now that $j_{3}=1$. Then by $\left(8^{\prime}\right)$ if $j_{2}=1$

$$
M=x y z y=-1 / 2 y^{2} z x \text {. }
$$

If $j_{2}=2$, then by (8), $\left(8^{\prime \prime \prime}\right)$

$$
M=x y y z y=-1 / 2 y^{2} x z y=-1 / 2\left((x z) \cdot y^{2}\right) y=x z y y y=0 .
$$


This completes the proof.

Now by the Corollary of the Lemma $G_{3}$ is nilpotent, so var $G_{3}$ has (2) and $\operatorname{var} G_{3}$ is one of the varieties (i)-(iii) from the conditions of Theorem 15, $\operatorname{var} G_{3} \supseteq \operatorname{var} G_{2}$, and therefore, in $\operatorname{var} G_{3}$,

$$
x y=y x, \quad x^{3}=J(x, y, z)=0, \quad x y z t=x y t z .
$$

Hence, in $V$ we have $x^{2} y z=x^{2} z y$. Linearizing this identity we obtain by commutativity in $V, x y z t=x y t z$. Now, as it was proved in [1], this implies $x y z t u=$ 0 . The theorem is proved.

Acknowledgement. This paper was written in Bedford College, London. Using this opportunity I would like to express my thanks to the staff of Mathematical Department, especially to Professor P. M. Cohn, Dr. W. Stephenson, Dr. W. Dicks, Mrs. E. Simpson for their hospitality and useful discussions.

\section{REFERENCES}

1. V. A. Artamonov, Chain varieties of linear algebras, Trudy Moskov. Mat. Obšč. 29 (1973), 51-77 = Trans. Moscow Math. Soc. 29 (1973), 49-76. 1212.

2. - On varieties of restricted Lie algebras, Sibirsk. Mat. Ž. 6 (1974), 1197-

3. S. Fajtlowicz, H. Subramanian and T. R. Sundaraman, Equationally precomplete rings, Notices Amer. Math. Soc. 18 (1971), 630. Abstract \#71T-A119.

4. T. R. Sundaraman, Lattice of precomplete varieties of rings, Indag. Math. 37 (1975), 144.

5. I. V. L'vov, On varieties of associative rings. I, Algebra i Logika 12 (1973), 269$297=$ Algebra and Logic 12 (1973), 150-167.

6. M. Osborn, Varieties of algebras, Advances in Math. 8 (1972), 163-370.

7. M. Nagata, Local rings, Interscience Tracts in Pure and Appl. Math., no. 13, Interscience, New York, 1962. MR 27 \#5790.

8. V. A. Artamonov, On finite algebras of prime dimension with no proper subalgebras, J. Algebra (to appear).

9. R. D. Schafer, An introduction to nonassociative algebras, Pure and Appl. Math., vol. 22, Academic Press, New York and London, 1966. MR 35 \#1643.

10. K. McCrimmon, Alternative algebras satisfying polynomial identities, J. Algebra 24 (1973), 283-292. MR 47 \#298.

11. N. Jacobson, Structure of rings, 2nd ed., Amer. Math. Soc. Colloq. Publ., vol. 37, Amer. Math. Soc., Providence, R. I., 1964. MR 36 \#5158.

12. K. McCrimmon, Finite power-associative division rings, Proc. Amer. Math. Soc. 17 (1966), 1173-1177. MR 34 \#4319. (Russian)

13. A. A. Nikitin, Almost altemative algebras, Algebra i Logika 13 (1974), 501-533.

14. K. A. Zevlakov and I. P. Shestakov, On local finiteness in sense of Shirshov, Algebra i Logika 12 (1973), 41-73. (Russian)

15. A. I. Siršov, On some non-associative nil-rings and algebraic algebras, Mat. Sb. 41 (83) (1957), 381-394. (Russian) MR 19, 727.

DEPARTMENT OF MECHANICS AND MATHEMATICS, MOSCOW UNIVERSITY, MOSCOW, 117234, USSR

DEPARTMENT OF MATHEMATICS, BEDFORD COLLEGE, REGENT'S PARK, LONDON, NW1 4NS, ENGLAND 